Document downloaded from:

http://hdl.handle.net/10251/154031

This paper must be cited as:

Sala, A. (2019). Stability analysis of LPV systems: Scenario approach. Automatica. 104:233237. https://doi.org/10.1016/j.automatica.2019.01.032

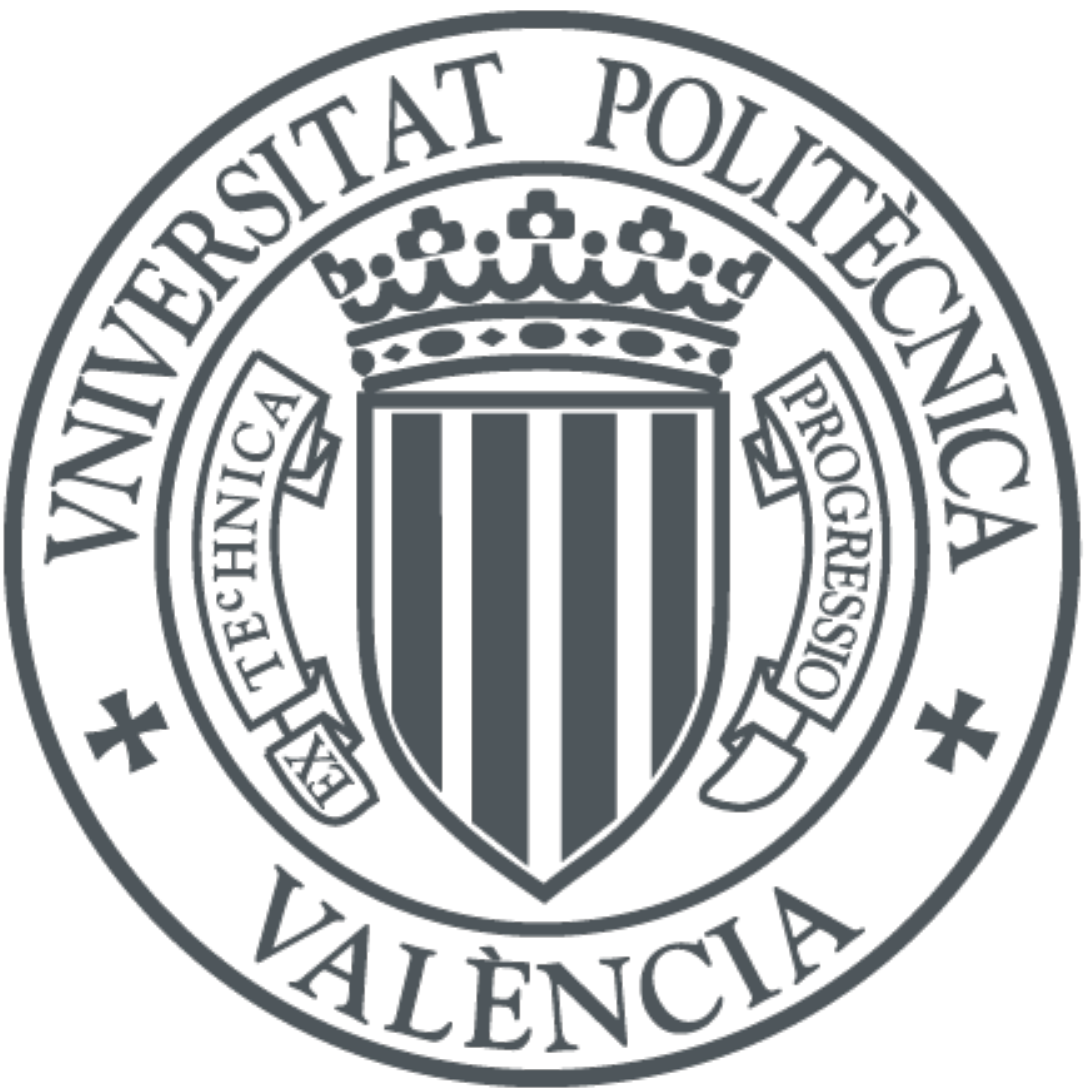

The final publication is available at

https://doi.org/10.1016/j.automatica.2019.01.032

Copyright Elsevier

Additional Information 


\title{
Stability Analysis of LPV Systems: Scenario Approach *
}

\author{
Antonio Sala \\ Inst. U. Automática Informática Industrial $\left(A I^{2}\right)$. Universitat Politècnica de València. \\ Cno de Vera s/n, E-46022 Valencia, Spain.
}

\begin{abstract}
This paper discusses a 'scenario' approach to prove decay-rate stability of discrete-time polytopic linear parameter-varying systems, dealing with sets of sequences of vertex models of different length. When all sequences have the same length, parametertrajectory dependent results in earlier literature are obtained as particular cases. The approach in this paper discusses 'classical' stability, without the need of probabilistic ingredients present in other scenario-based ideas in literature. A numerical example shows that the proposal achieves a sensible tradeoff between proven performance and computing requirements.
\end{abstract}

Key words: linear parameter-varying systems, discrete-time systems, parameter-dependent Lyapunov function

\section{Introduction}

The work [15] presents a multi-step generalisation of earlier results [4] on parameter-dependent Lyapunov functions for discrete-time LPV systems. In fact, the proposal in [15] is related to prior work, such as the pathdependent Lyapunov function in [10, Thm 9.], $k$-sample variation in $[8,5]$ or the multi-instant approaches in [14]. These approaches propose considering increments of a Lyapunov functions $V(x(k+N))-V(x(k))$ with $N>1$. The multi-instant approach has two main advantages [5]: first, most conditions for a given $N$ can be proved to be less conservative than these for $N / 2, N / 3, \ldots$ and, second, if a system is asymptotically stable, any strictly positive-definite quadratic function, say, $V(x)=x^{T} x$, will fulfill $V(x(k+N))-V(x(k))<0$ for long enough $N$.

The basic drawback of multi-instant approaches is that the number of linear matrix inequality (LMI) constraints exponentially increases with the horizon $N$. Scenario approaches have appeared in literature; in them, only a subset of the vertex or scheduling parameter trajectories is analyzed with substantially lower computational cost. This is the main motivation of the present work.

Probabilistic approaches to robust control assess the risk of constraint violation $[3,1,6]$; these 'risky' approaches will not be considered this work. Alternatively, the paper

\footnotetext{
* This paper was not presented at any IFAC meeting. The author is grateful to Ministerio de Economia (Spain) and European Union, grant DPI2016-81002-R.

Email address: asala@isa.upv.es (Antonio Sala).
}

[12] presents a scenario approach in which probabilisticcomplete scenarios provide mean-square stability and recursive feasibility guarantees for model predictive control of Markov-jump linear systems, with no 'risk' of divergence/infeasibility, without requiring the full $r^{N}$ scenario needed in [11] for the same problem.

The objective of this work is discussing the analogous of [12] for a LPV formulation, with a standard worst-case (i.e., not probabilistic) geometric decay-rate guarantee. Basically, a subset of vertex sequences of possibly different length will be enough to guarantee stability. In this way, stability analysis results of [15] and related works will be a particular case of the proposal here, in the same way as results in [11] were a particular case of [12].

\section{Preliminaries and notation}

Consider a discrete-time LPV system:

$$
x(k+1)=A(\xi(k)) x(k), \quad k \in \mathbb{N} \cup\{0\}
$$

where $x \in \mathbb{R}^{n}$ is the state vector, and $\xi(k)$ is a vector of time-varying scheduling parameters such that

$$
A(\xi(k))=\sum_{i \in \mathcal{I}}^{r} \xi_{i}(k) A_{i} \quad \forall k \geq 0
$$

where $A_{i}$ are denoted as vertex models, $\mathcal{I}:=\{1, \ldots, r\}$, and the scheduling parameters $\xi(k)$ lie in the standard simplex $\Delta:=\left\{\psi \in \mathbb{R}^{r}: \sum_{i \in \mathcal{I}} \psi_{i}=1, \forall i \in \mathcal{I} \xi_{i} \geq\right.$ $0\}$. An amplification factor $\sigma$ will be defined as $\boldsymbol{\sigma}:=$ $\max _{i \in \mathcal{I}} \bar{\sigma}\left(A_{i}\right)$ where $\bar{\sigma}$ denotes the maximum singular 
value. A direct consequence of the definition of $\boldsymbol{\sigma}$, denoting the Euclidean norm as $\|\cdot\|_{2}$, is the fact that

$$
\|x(k+\eta)\|_{2} \leq \boldsymbol{\sigma}^{\eta}\|x(k)\|_{2} .
$$

Definition 1 System (1) achieves a rate ${ }^{1} \gamma>0$ if, for any parameter trajectory $\xi: \mathbb{N} \rightarrow \Delta$, for any $x(0)$, there exists $c>0$ such that

$$
\|x(k)\|_{2} \leq c \cdot \gamma^{k} \cdot\|x(0)\|_{2}
$$

We will denote as $\gamma^{*}$ the minimum $\gamma$ for which (4) holds.

Given system (1), we will define its associated switching system as the LPV system fulfilling (1) and (2) with the additional constraint of $\xi \in\{0,1\}^{r}$. It is well known [9] that stability of this switching system is equivalent to stability of the original LPV system (1), as the set of trajectories of (1) coincides with the convex hull of these from the switching system. Hence, on the sequel, we need only studying $x(k+1)=A_{i_{k}} x(k), i_{k} \in \mathcal{I}$, for all vertex trajectories $\left(i_{0}, i_{1}, \ldots\right) \in \mathcal{I}^{\infty}$, with $\mathcal{I}^{\infty}$ being the set of all infinite-length sequences of elements in $\mathcal{I}$.

Numerical computations with infinite-length vertex sequences are not possible, so finite-length ones will be introduced. Boldface notation will define an ordered sequence of vertex indices, $\mathbf{i}:=\left(i_{0}, \ldots, i_{N-1}\right)$, with $i_{k} \in \mathcal{I}$, $N$ being the sequence length, also referred to as 'horizon'. The above will be shorthanded to $\mathbf{i} \in \mathcal{I}^{N}$, where $\mathcal{I}^{N}$ denotes the set of all sequences of length $N$, with cardinality, i.e., number of sequences, $\operatorname{card}\left(\mathcal{I}^{N}\right)=r^{N}$. The set of all finite-length sequences will be denoted by $\mathcal{I}^{*}:=\bigcup_{N \in \mathbb{N}} \mathcal{I}^{N}$. When several sequences of different lentgh are involved, notation $N_{\mathbf{i}}$ will denote the length of sequence $\mathbf{i}$, with $N_{\mathbf{i}}:=\infty$ for $\mathbf{i} \in \mathcal{I}^{\infty}$.

Given an initial condition $x(0)$ and a particular vertex sequence $\mathbf{i} \in \mathcal{I}^{*} \cup \mathcal{I}^{\infty}$, the trajectory of the switching system under $\mathbf{i}$ will be defined, for $0 \leq k \leq N_{\mathbf{i}}-1$, as:

$$
x_{\mathbf{i}}(k+1):=A_{i_{k}} x_{\mathbf{i}}(k)=A_{i_{k}} \ldots A_{i_{0}} x(0)
$$

understanding $x_{\mathbf{i}}(0):=x(0)$. The switching system achieving rate $\gamma$ will be understood as verifying (4) replacing $x(k)$ by $x_{\mathbf{i}}(k)$, for all $\mathbf{i} \in \mathcal{I}^{\infty}$, and that is equivalent to the LPV (1) achieving rate $\gamma$, as above discussed.

In order to shorthand expressions like (5), given a finite sequence $\mathbf{i} \in \mathcal{I}^{*}$, we will introduce the product notation:

$$
A_{\mathbf{i}}:=A_{i_{N-1}} A_{i_{N-2}} \ldots A_{i_{0}}
$$

Last, $P \succ 0$ and $P \preceq 0$ will denote positive-definite and negative-semidefinite matrices, respectively.

\footnotetext{
1 If a system achieves a rate $\gamma<1$, it is usually said that it is stable with discrete decay rate $\gamma$. Trivially from (3), (1) achieves any rate $\gamma \geq \boldsymbol{\sigma}$, motivating Corollary 1 later on.
}

Theorem 1 [15] If, given $\gamma>0$, for all $\mathbf{i} \in \mathcal{I}^{N}, \mathbf{j} \in \mathcal{I}^{N}$, there exist positive definite matrices $P_{\mathbf{i}} \succ 0$ such that the $r^{2 N}$ linear matrix inequalities

$$
A_{\mathbf{i}}^{T} P_{\mathbf{j}} A_{\mathbf{i}}-\gamma^{2 N} P_{\mathbf{i}} \preceq 0
$$

hold, then the LPV system (1) achieves a rate $\gamma$. Conversely, if (1) achieves a rate $\gamma^{*}$, for any $\gamma>\gamma^{*}$, horizon $N \geq \log c /\left(\log \gamma-\log \gamma^{*}\right)$ makes conditions (7) feasible.

Proof: The first statement is a straightforward decayrate generalisation of [15, Th. 1] and it will be a particular case of the more general Theorem 2, presented in next section. Regarding the second statement, (4) implies that, for all $\mathbf{i} \in \mathcal{I}^{N}$, for all $N \geq 1, A_{\mathbf{i}}^{T} A_{\mathbf{i}}-c^{2}\left(\gamma^{*}\right)^{2 N} I \preceq 0$. If there exists $N$ such that $c^{2}\left(\gamma^{*}\right)^{2 N} \leq \gamma^{2 N}$, the assertion is proved; such $N$ always exists if $\gamma>\gamma^{*}$, trivial manipulations result in the inequality in the statement.

The objective of this communique is achieving comparable results to Theorem 1 with a significantly lower number of LMIs than the $r^{2 N}+r^{N}$ needed in it.

\section{Main result: scenario approach}

Definition 2 A 'scenario', denoted by $\mathbf{\Theta}$, will be defined as a finite set of finite-length sequences, i.e., $\Theta \subset \mathcal{I}^{*}$. Each individual sequence may have a different horizon, the scenario horizon will be defined as $N_{\Theta}:=\max _{\mathbf{i} \in \Theta} N_{\mathbf{i}}$.

Definition 3 The leading fragment of $\mathbf{i} \in \mathcal{I}^{*} \cup \mathcal{I}^{\infty}$ with length $N^{\prime} \leq N_{\mathbf{i}}$ will be defined as the sequence $\left(i_{0}, \ldots, i_{N^{\prime}-1}\right)$ comprised of the first $N^{\prime}$ elements of $\mathbf{i}$. A scenario $\Theta$ will be said to be complete if, for every sequence $\mathbf{j} \in \mathcal{I}^{\infty}$, there exists a sequence ${ }^{2}$ in $\boldsymbol{\Theta}$ that is a leading fragment of $\mathbf{j}$.

For instance, with $r=2,\{(1,1),(1,2), 2\}$ is complete, whereas the scenario $\boldsymbol{\Theta}:=\{(1,1,1),(1,2), 2\}$ is not, as $\mathbf{j}=(1,1,2, \ldots)$ does not have any leading fragment in $\boldsymbol{\Theta}$ : indeed, $(1) \notin \boldsymbol{\Theta},(1,1) \notin \boldsymbol{\Theta},(1,1,2) \notin \boldsymbol{\Theta}$. In Section 4 , scenarios in (16) are, too, complete. The scenario $\mathcal{I}^{N}$, denoted in the sequel as the full scenario of horizon $N$, is, evidently, complete; actually, Theorem 2, the main result of this note, reduces to Theorem 1 if $\boldsymbol{\Theta}=\mathcal{I}^{N}$.

Theorem 2 Consider a complete scenario $\boldsymbol{\Theta}$, and $\gamma>$ 0 . If, for all $\mathbf{i} \in \boldsymbol{\Theta}$, for all $\mathbf{j} \in \boldsymbol{\Theta}$, there exist positive definite matrices $P_{\mathbf{i}} \succ 0$ such that

\footnotetext{
$\overline{2}$ If such a sequence in $\Theta$ is unique, the scenario will be called non-redundant; these scenarios can be equivalently considered as tree structures [12]. Such tree structure is not formally needed in the developments here, but, nevertheless, non-redundant scenarios are recommended, to avoid inducing conservatism due to unneeded LMI constraints in Theorem 2 below (details omitted for brevity).
} 


$$
A_{\mathbf{i}}^{T} P_{\mathbf{j}} A_{\mathbf{i}}-\gamma^{2 N_{\mathbf{i}}} P_{\mathbf{i}} \preceq 0
$$

the LPV system (1) achieves a rate $\gamma$.

Proof: As discussed in Section 2, by convexity argumentations, a given rate for (1) is guaranteed if it is proven for its associated switching system. Thus, in the rest of the proof, states and trajectories will refer to the latter.

Completeness of $\boldsymbol{\Theta}$ ensures that any vertex trajectory of the switching system will start with a vertex sequence in $\boldsymbol{\Theta}$. In other words, using notation in (5), for all $\mathbf{t} \in \mathcal{I}^{\infty}$, there exists $\mathbf{i} \in \boldsymbol{\Theta}$ such that, $x_{\mathbf{t}}\left(N_{\mathbf{i}}\right)=x_{\mathbf{i}}\left(N_{\mathbf{i}}\right)$. Thus, under the completeness assumption, if (8) is feasible, for any trajectory $x_{\mathbf{t}}(k)$ of the switching system (starting in an arbitrary $x(0))$, for all $\mathbf{j}$ in $\boldsymbol{\Theta}$, we have:

$$
x_{\mathbf{t}}\left(N_{\mathbf{i}}\right)^{T} P_{\mathbf{j}} x_{\mathbf{t}}\left(N_{\mathbf{i}}\right) \leq \gamma^{2 N_{\mathbf{i}}} \cdot x(0)^{T} P_{\mathbf{i}} x(0)
$$

which can be equivalently rewritten as

$$
\max _{\mathbf{j} \in \boldsymbol{\Theta}} x_{\mathbf{t}}\left(N_{\mathbf{i}}\right)^{T} P_{\mathbf{j}} x_{\mathbf{t}}\left(N_{\mathbf{i}}\right) \leq \gamma^{2 N_{\mathbf{i}}} \cdot x(0)^{T} P_{\mathbf{i}} x(0)
$$

Maximising the right-hand side of the above inequality over all sequences in $\boldsymbol{\Theta}$, we can assert:

$$
\max _{\mathbf{j} \in \mathbf{\Theta}} x_{\mathbf{t}}\left(N_{\mathbf{i}}\right)^{T} P_{\mathbf{j}} x_{\mathbf{t}}\left(N_{\mathbf{i}}\right) \leq \gamma^{2 N_{\mathbf{i}}} \max _{\mathbf{j} \in \mathbf{\Theta}} x(0)^{T} P_{\mathbf{j}} x(0)
$$

In summary, considering the Lyapunov-like function

$$
V(x):=\max _{\mathbf{j} \in \mathbf{\Theta}} x^{T} P_{\mathbf{j}} x .
$$

expression (9) proves that there exists a finite time instant in the future $\nu_{1}=N_{\mathbf{i}} \leq N_{\Theta}$ such that

$$
V\left(x_{\mathbf{t}}\left(\nu_{1}\right)\right) \leq \gamma^{2 \nu_{1}} V(x(0))
$$

Now, let us denote as $\mathbf{t}^{\prime}:=\left(t_{\nu_{1}}, t_{\nu_{1}+1}, t_{\nu_{1}+2}, \ldots\right) \in \mathcal{I}^{\infty}$ the infinite-length trailing fragment of sequence $\mathbf{t}$ obtained by removing its first $\nu_{1}$ leading elements. Repeating the argumentation with $\mathbf{t}^{\prime}$ (i.e., conceptually, setting the time origin at $\left.\nu_{1}\right)$, completeness of $\boldsymbol{\Theta}$ ensures, again, that there exists a finite $\nu_{2}$ such that:

$$
V\left(x_{\mathbf{t}}\left(\nu_{2}+\nu_{1}\right)\right) \leq \gamma^{2 \nu_{2}} V\left(x_{\mathbf{t}}\left(\nu_{1}\right)\right)
$$

and, hence, using (11) at the right-hand side,

$$
V\left(x_{\mathbf{t}}\left(\nu_{2}+\nu_{1}\right)\right) \leq \gamma^{2\left(\nu_{2}+\nu_{1}\right)} V(x(0))
$$

As, actually, the argumentation can be repeated ad infinitum, that proves that there exist a sequence of 'Lyapunov sampling instants', $\Upsilon:=\left(\eta_{0}, \eta_{1}, \eta_{2}, \ldots\right)$, being $\eta_{0}=0, \eta_{m}:=\sum_{j=1}^{m} \nu_{j}, m \in \mathbb{N}$, such that

$$
V\left(x_{\mathbf{t}}\left(\eta_{m}\right)\right) \leq \gamma^{2 \eta_{m}} V(x(0)) \quad \forall m \geq 0
$$

and the 'dwell-time' verifies $\eta_{m+1}-\eta_{m}=\nu_{m+1} \leq N_{\Theta}$.

Let us now prove that (4) holds, both in sampling instant and in intermediate ones. Note that $V(x)$ fulfills

$$
\mu_{l} \cdot\|x\|_{2}^{2} \leq V(x) \leq \mu_{u} \cdot\|x\|_{2}^{2}
$$

where

$$
\mu_{l}=\min _{\mathbf{i} \in \boldsymbol{\Theta}} \min \operatorname{eig}\left(P_{\mathbf{i}}\right), \quad \mu_{u}=\max _{\mathbf{i} \in \mathbf{\Theta}} \max \operatorname{eig}\left(P_{\mathbf{i}}\right),
$$

being $\operatorname{eig}(\cdot)$ the set of eigenvalues. As $\mu_{l}>0,(14)$ entails

$$
\left\|x_{\mathbf{t}}\left(\eta_{i}\right)\right\|_{2} \leq \sqrt{\frac{\mu_{u}}{\mu_{l}}} \gamma^{\eta_{i}}\|x(0)\|_{2} .
$$

From (3), we can ensure that, for all $\mathbf{t} \in \mathcal{I}^{\infty}$, intermediate states $x_{\mathbf{t}}(k)$, with $\eta_{m} \leq k \leq \eta_{m+1}$ fulfill:

$$
\begin{aligned}
& \left\|x_{\mathbf{t}}(k)\right\|_{2} \leq \sqrt{\frac{\mu_{u}}{\mu_{l}}} \boldsymbol{\sigma}^{k-\eta_{m}} \gamma^{\eta_{m}}\|x(0)\|_{2} \\
= & \sqrt{\frac{\mu_{u}}{\mu_{l}}} \boldsymbol{\sigma}^{k-\eta_{m}} \gamma^{\eta_{m}-k} \gamma^{k}\|x(0)\|_{2}=\sqrt{\frac{\mu_{u}}{\mu_{l}}}\left(\frac{\boldsymbol{\sigma}}{\gamma}\right)^{k-\eta_{m}} \gamma^{k}\|x(0)\|_{2}
\end{aligned}
$$

so, if $\gamma \geq \boldsymbol{\sigma}$, we can bound $\left(\frac{\boldsymbol{\sigma}}{\gamma}\right)^{k-\eta_{m}} \leq 1$, and otherwise we can bound $\left(\frac{\boldsymbol{\sigma}}{\gamma}\right)^{k-\eta_{m}} \leq\left(\frac{\boldsymbol{\sigma}}{\gamma}\right)^{N_{\Theta}}$. Thus, if we denote:

$$
c:=\sqrt{\frac{\mu_{u}}{\mu_{l}}} \cdot \max \left(1,\left(\frac{\boldsymbol{\sigma}}{\gamma}\right)^{N_{\Theta}}\right)
$$

we have $\left\|x_{\mathbf{t}}(k)\right\|_{2} \leq c \gamma^{k}\|x(0)\|_{2}$ for all $\mathbf{t} \in \mathcal{I}^{\infty}$, for all $k \in \mathbb{N}$, so we proved the rate $\gamma$ for the switched system and, henceforth, for the original LPV system (1).

Corollary 1 Let us denote as $\gamma_{\Theta}^{*}$ the minimum $\gamma$ so that conditions in Theorem 2 are feasible for a scenario $\boldsymbol{\Theta}$, and $\bar{\gamma}_{\text {min }}:=\max _{\mathbf{i} \in \Theta} \max \left(\operatorname{abs}\left(e i g\left(A_{\mathbf{i}}\right)\right)\right)^{1 / N_{\mathbf{i}}}$, and $\bar{\gamma}_{s v}:=$ $\max _{\mathbf{i} \in \boldsymbol{\Theta}} \bar{\sigma}\left(A_{\mathbf{i}}\right)^{1 / N_{\mathrm{i}}}$. Then, $\bar{\gamma}_{\text {min }} \leq \gamma^{*} \leq \gamma_{\boldsymbol{\Theta}}^{*} \leq \bar{\gamma}_{s v} \leq \boldsymbol{\sigma}$.

Proof: It is well known that the system's rate $\gamma^{*}$ cannot be smaller than the one associated to the dominant eigenvalue of any transition matrix $A_{\mathbf{i}}$, thus $\bar{\gamma}_{\text {min }} \leq$ $\gamma^{*}$. Proof of Theorem 2 ensures $\gamma^{*} \leq \gamma_{\Theta}^{*}$. Now, in an analogous way to (3), setting $P=I$, the inequality $A_{\mathbf{i}}^{T} A_{\mathbf{i}}-\kappa_{\mathbf{i}}^{2} I \preceq 0$ holds for $\kappa_{\mathbf{i}}$ greater than or equal to the largest singular value of $A_{\mathbf{i}}$, denoted as $\bar{\sigma}\left(A_{\mathbf{i}}\right)$; the maximum over $\mathbf{i} \in \mathbf{\Theta}$ justifies $\gamma_{\Theta}^{*} \leq \bar{\gamma}_{s v}$. Elementary submultiplicative singular-value properties prove $\bar{\gamma}_{s v} \leq \boldsymbol{\sigma}$.

Discussion. Note that, considering $\gamma$ as decision variable, Theorem 2 is a generalised eigenvalue problem; Corollary 1 sets up initial bounds for a bisection algorithm to minimise $\gamma$. The number of LMIs is $s(s+1)$, being $s:=\operatorname{card}(\boldsymbol{\Theta})$. The number of decision variables $(\mathrm{DV})$ is $\frac{1}{2} n(n+1) * s$.

Note also that $V(x)$ in (10) does only depend on the state, instead of the parameter-memorised version of [15] or other trajectory-dependent proposals in literature; similar argumentations to eliminate past parameter trajectory dependence appear in, for instance, [13, p. 13] 
or [2, Thm. 3]. Note that, formally, $V(x)$ is not a Lyapunov function as it only decreases 'from time to time', at irregularly-spaced instants (trajectory-dependent). Note also that possibly less conservative options for the maximum-of-quadratics (10) may be posed, using S-procedure multipliers [7]; however, such multipliers render the problem into non-convex bilinear matrix inequalities, so the approach will be pursued no further.

\section{Scenario generation/growth.}

The above results consider $\Theta$ as given, a priori. Finding the lowest-cardinality scenario proving a given rate by brute force is not an option, as the number of scenarios grows hugely with horizon $N$; thus, this section will present some heuristics guiding the scenario choice.

First, Theorem 2 reduces conservatism as scenario horizon gets longer, in a particular way discussed next. Square bracket notation $[\mathbf{i}, \mathbf{j}, \mathbf{k}, \ldots]$ will stand for the concatenation of sequences, with resulting length $N_{\mathbf{i}}+N_{\mathbf{j}}+N_{\mathbf{k}}+\ldots ;$ for instance, given sequences $\mathbf{i}$ and $\mathbf{j}$, we let $[\mathbf{i}, \mathbf{j}]:=\left(i_{0}, i_{1}, \ldots, i_{N_{\mathbf{i}}-1}, j_{0}, j_{1}, \ldots, j_{N_{\mathbf{j}}-1}\right)$.

Corollary 2 Assume that conditions in Theorem 2 are feasible for given $\boldsymbol{\Theta}$ and $\gamma$. Consider any sequence $\mathbf{j}$ built as the concatenation of $M$ arbitrary sequences in $\mathbf{\Theta}$, i.e., $\mathbf{j}=\left[\mathbf{i}^{[1]}, \ldots, \mathbf{i}^{[M]}\right]$, with $\mathbf{i}^{[q]} \in \boldsymbol{\Theta}, q=1, \ldots$, M. Then, the $L M I$

$$
A_{\mathbf{j}}^{T} P_{\mathbf{h}} A_{\mathbf{j}}-\gamma^{2 N_{\mathbf{j}}} P_{\mathbf{i}^{[1]}} \preceq 0
$$

is feasible for any $\mathbf{h} \in \mathbf{\Theta}$.

Proof: As $A_{\mathbf{j}}=A_{\mathbf{i}^{[M]}} \ldots A_{\mathbf{i}^{[2]}} A_{\mathbf{i}^{[1]}}$, and $N_{\mathbf{j}}=\sum_{p=1}^{M} N_{\mathbf{i}^{[p]}}$, we can state that

$$
\begin{gathered}
A_{\mathbf{j}}^{T} P_{\mathbf{h}} A_{\mathbf{j}}=A_{\mathbf{i}^{[1]}}^{T} \ldots A_{\mathbf{i}^{[M-1]}}^{T} A_{\mathbf{i}^{[M]}}^{T} P_{\mathbf{h}} A_{\mathbf{i}^{[M]}} \ldots A_{\mathbf{i}^{[2]}} A_{\mathbf{i}^{[1]}} \\
\leq \gamma^{2 N_{\mathbf{i}[M]}} \cdot A_{\mathbf{i}^{[1]}}^{T} \ldots A_{\mathbf{i}^{[M-1]}}^{T} P_{\left.\mathbf{i}^{[M]}\right]} A_{\mathbf{i}^{[M-1]}} \ldots A_{\mathbf{i}^{[2]} A_{\left.\mathbf{i}^{[1]}\right]}} \leq \\
\vdots \\
\leq\left(\gamma^{\left.2 \sum_{p=1}^{M-1} N_{\mathbf{i}^{[p]}}\right)} A_{\mathbf{i}^{[1]}}^{T} P_{\mathbf{i}^{[2]}} A_{\mathbf{i}^{[1]}} \leq\left(\gamma^{2 N_{\mathbf{j}}}\right) P_{\mathbf{i}^{[1]}}\right.
\end{gathered}
$$

Corollary 3 If, given $\gamma$, conditions in Theorem 2 are feasible for $\boldsymbol{\Theta}$, so they are for any complete scenario $\boldsymbol{\Theta}^{\prime}$ whose sequences can be expressed as a concatenation of those in $\boldsymbol{\Theta}$. Hence, $\gamma_{\boldsymbol{\Theta}^{\prime}}^{*} \leq \gamma_{\boldsymbol{\Theta}}^{*}$.

Proof: For any $\mathbf{j} \in \mathbf{\Theta}^{\prime}$, partitioned as in Corollary 2, the corollary proves that the choice $P_{\mathbf{j}}:=P_{\mathbf{i}}{ }^{[1]}$ renders a feasible solution for conditions in Theorem 2 with $\Theta^{\prime}$.

Remark: the above corollary can be used to prove feasibility of the 'product' scenario $\Theta^{M}$ defined as the one whose sequences are the concatenation of exactly $M$ sequences in $\boldsymbol{\Theta}$, with $\operatorname{card}\left(\boldsymbol{\Theta}^{M}\right)=\operatorname{card}(\boldsymbol{\Theta})^{M}$; full-scenario is the particular case in [5] proving that $\gamma_{\mathcal{I}^{(\kappa N)}}^{*} \leq \gamma_{\mathcal{I}^{N}}^{*}, \kappa \in \mathbb{N}$. For brevity, details are omitted.
AlgORITHM 1

(1) Set $\boldsymbol{\Theta}_{[1]}=\mathcal{I}$. Set $k=1, \underline{\gamma}=0, \widetilde{\gamma}=+\infty$

(2) Compute $\gamma_{\Theta_{[k]}}^{*}$, by minimising $\gamma$ in Th. 2.

(3) Compute lower bound $\bar{\gamma}_{\min }$ (Cor. 1) for scenario $\Theta_{[k]}$.

(4) Let $\underline{\gamma}=\max \left(\underline{\gamma}, \bar{\gamma}_{\text {min }}\right), \widetilde{\gamma}=\min \left(\widetilde{\gamma}, \gamma_{\mathbf{\Theta}_{[k]}}^{*}\right)$.

(5) If $\widetilde{\gamma}-\underline{\gamma}<\varepsilon$, END; we proved $\gamma^{*} \in[\underline{\gamma}, \widetilde{\gamma}]$.

(6) Find the sequences yielding active constraints:

$$
\mathbf{i}^{\prime}:=\arg \max _{\mathbf{i} \in \boldsymbol{\Theta}_{[k]}} \max _{\mathbf{j} \in \mathbf{\Theta}_{[k]}} \operatorname{eig}\left(A_{\mathbf{i}}^{T} P_{\mathbf{j}} A_{\mathbf{i}}-\gamma_{\mathbf{\Theta}}^{*} P_{\mathbf{i}}\right) .
$$

(7) Branch them either by incrementing its horizon one unit, or by appending every sequence of $\boldsymbol{\Theta}_{[k]}$; denote the resulting scenario as $\boldsymbol{\Theta}_{[k+1]}$. Let $k=k+1$.

(8) Go to step 2.

*In step $5, \varepsilon>0$ is a small enough termination tolerance.

Scenario branching. Corollary 3 proves feasibility of a scenario obtained from 'branching' (in the tree-like analogy of [12]) a complete and non-redundant $\boldsymbol{\Theta}$, replacing one of its sequences by the concatenation of it with each of the sequences in $\Theta$, resulting in a new scenario $\boldsymbol{\Theta}^{\prime}$ with $\operatorname{card}\left(\boldsymbol{\Theta}^{\prime}\right)=2 \cdot \operatorname{card}(\boldsymbol{\Theta})-1$. Alternatively, replacing $\mathbf{i}$ by a set of $r$ new sequences $[\mathbf{i}, j], j \in \mathcal{I}$, yields a new complete scenario $\boldsymbol{\Theta}^{\prime}$, increasing the horizon by 1 , and the cardinality by $r-1$. This branching is convenient to avoid large increases in cardinality and horizon, and it has been used in the examples, but it does not guarantee monotonic performance improvement $\gamma_{\Theta^{\prime}}^{*} \leq \gamma_{\Theta}^{*}$ as the first option does.

Scenario growth algorithm. A sensible use of Theorem 2 will, in most cases, start with $\Theta=\mathcal{I}$. In order to approach the minimum rate $\gamma^{*}$ for $(1)$, longer scenarios should be sought, trying to avoid to the full scenario. The above-discussed branching can be used for such purpose; however, selecting a sequence to branch needs some thought. Inspired in Corollary 1, the sequence to branch may be chosen as $\mathbf{i}^{\prime}:=\arg \max _{\mathbf{i} \in \Theta} \bar{\sigma}\left(A_{\mathbf{i}}\right)^{1 / N_{\mathbf{i}}}$, i.e., any of those yielding $\bar{\gamma}_{s v}$, to build an scenario with low singular value bound. As a possibly better alternative, given $\Theta$ and its best provable rate $\gamma_{\Theta}^{*}$, the sequences to branch may be selected by choosing the ones associated to active LMI constraints. Indeed, the latter branching approach has been integrated into Algorithm 1, proposing an iterative scenario growth mechanism until a termination criteria is met (the exact decay $\gamma^{*}$ of system (1) is found if the smallest $\gamma_{\Theta_{[k]}^{*}}^{*}$ coincides with the largest eigenvalue-based bound $\bar{\gamma}_{\text {min }}$ ).

\section{Example}

Consider a LPV system with vertex matrices given by ${ }^{3}$ : $A_{1}=\left(\begin{array}{cc}0.9520 & 0.0936 \\ -0.9358 & 0.8584\end{array}\right), A_{2}=\left(\begin{array}{cc}0.9996 & 0.0824 \\ -0.0082 & 0.6699\end{array}\right)$,

${ }_{3} A_{1}$ and $A_{2}$ are the same as in [15, Ex. 1]. 


$$
A_{3}=\left(\begin{array}{cc}
0.9 & 0.1 \\
-0.1 & 0.6
\end{array}\right)
$$

The minimum feasible rate $\gamma_{\boldsymbol{\Theta}}^{*}$, computed by bisection (tolerance $<10^{-6}$ ) with full scenarios, i.e., applying Theorem 1, as well as the number of involved sequences, LMIs and computation time ${ }^{4}$ are:

\begin{tabular}{c|cccc}
\hline Th.1:[15] $N_{\Theta}$ & 1 & 2 & 3 & 4 \\
\hline$\gamma_{\Theta}^{*}$ & 1.00362 & 0.99867 & 0.99754 & 0.99754 \\
Num. seq. & 3 & 9 & 27 & 81 \\
LMIs|time(s) & $12 \mid 4.8$ & $90 \mid 9.4$ & $756 \mid 38$ & $6642 \mid 340$ \\
The gray column, with $\gamma_{\Theta}^{*} \geq 1$, highlights that stability can- \\
not be proven with the chosen scenario.
\end{tabular}

As $\max \left(\left|\operatorname{eig}\left(A_{2}\right)\right|\right)=0.99754$, and $\gamma_{\mathcal{I}^{3}}^{*}=0.99754$ we have $\gamma_{\Theta}^{*}=\bar{\gamma}_{\min }$, hence we have found the actual $\gamma^{*}$ of the LPV system, so there is no surprise in numerically finding that scenarios with larger horizon do not improve over this result.

Now, starting with $\boldsymbol{\Theta}_{[1]}:=\mathcal{I}=\{1,2,3\}$ and using Algorithm 1 , we get the following scenarios in the three first iterations ${ }^{5}$, and the numerical results below:

$$
\begin{aligned}
\boldsymbol{\Theta}_{[1]} & =\{\underline{(1)}, \overline{(2),},(3)\}, \\
\boldsymbol{\Theta}_{[2]} & =\{\overline{(1,1)}, \overline{(1,2)}, \overline{(1,3)}, \overline{(2,1)}, \overline{(2,2)}, \overline{(2,3)},(3)\}, \\
\boldsymbol{\Theta}_{[3]} & =\{\overline{(1,1,1)}, \overline{(1,1,2)}, \overline{(1,1,3)}, \overline{(1,2)},(1,3),(2,1), \\
& \overline{(2,2,1)}, \overline{(2,2,2)}, \overline{(2,2,3)},(2,3),(3)\} \quad(16)
\end{aligned}
$$

\begin{tabular}{|c|c|c|c|c|}
\hline Alg. 1: $N_{\Theta}$ & 1 & 2 & 3 & 4 \\
\hline$\gamma_{\Theta}^{*}$ & 1.00362 & 0.998667 & 0.99754 & 0.99754 \\
\hline Num. seq. & $3 \Theta_{[1]}$ & $7 \Theta_{[2]}$ & $11 \Theta_{[3]}$ & 13 \\
\hline LMIs|time(s) & $12 \mid 4.8$ & $56 \mid 5.4$ & $132 \mid 7.8$ & $182 \mid 9.3$ \\
\hline
\end{tabular}

So, 132 LMIs (instead of 756 with $\mathcal{I}^{3}$ ) suffice to find $\gamma^{*}$.

From Theorem 1, a large enough horizon will prove any $\gamma>\gamma^{*}$ with $P=I$; thus, more conservative LMIs were, too, set up, with a common $P_{\mathbf{i}} \equiv P$. Now, Algorithm 1 finds the optimal rate $\gamma^{*}=0.99754$ with a scenario with maximum horizon 19, comprising a total of 87 sequences (88 LMIs, 5.3 seconds computing time). The full scenario $\mathcal{I}^{19}$ has $1+3^{19}=1.16 \cdot 10^{9}$ LMIs (intractable). The scenario $\mathcal{I}^{6}$ (729 sequences) can just prove a rate of 1.0032 with common $P$, with 39 s of computation time.

\footnotetext{
4 Code was not optimised for speed, so execution time is just illustrative when compared to other scenarios. Used software: yalmpip R20180817, sedumi 1.3, and Matlab R2018a, running on an i7-4790K CPU with default options. The number of decision variables is three times the number of sequences, except in the quadratic Lyapunov function case, in which it is equal to 3 , regardless of the scenario.

5 Underline highlights 'branched' sequences to generate the next scenario, overline marks the resulting 'child' sequences. As $\gamma_{\Theta_{[3]}}^{*}=\gamma^{*}$ there is no need of branching to depth 4, tested only for illustration (Algorithm 1 would stop with $\boldsymbol{\Theta}_{[3]}$ ).
}

\section{Conclusions}

This paper generalises some multi-step and trajectorydependent Lyapunov functions for stability analysis of LPV/switching systems in earlier literature: instead of considering a full scenario, with exponential complexity as the horizon increases, results in this work can use a set of sequences of different length. Expanding an standard 'horizon 1' scenario with a suitable branching, the results can prove the same performance than earlier 'full scenario' literature, with significantly lower computational cost.

\section{References}

[1] Teodoro Alamo, Roberto Tempo, Amalia Luque, and Daniel R. Ramirez. Randomized methods for design of uncertain systems: Sample complexity and sequential algorithms. Automatica, 52:160 - 172, 2015.

[2] Carlos Ariño, Antonio Sala, Emilio Pérez, Fernando Bedate, and Andres Querol. Asymptotically exact stabilisation for constrained discrete Takagi-Sugeno systems via setinvariance. Fuzzy Sets and Systems, 316:117-138, 2017.

[3] Giuseppe C Calafiore and Marco C Campi. The scenario approach to robust control design. IEEE Transactions on Automatic Control, 51(5):742-753, 2006.

[4] Jamal Daafouz and Jacques Bernussou. Parameter dependent Lyapunov functions for discrete time systems with time varying parametric uncertainties. Systems $\&$ control letters, 43(5):355-359, 2001.

[5] Thierry Marie Guerra, Alexandre Kruszewski, and Jimmy Lauber. Discrete Takagi-Sugeno models for control: Where are we? Annual Reviews in control, 33(1):37-47, 2009.

[6] Manuel A Hernández-Mejías and Antonio Sala. Reliability and time-to-failure bounds for discrete-time constrained Markov jump linear systems. International Journal of Robust and Nonlinear Control, 27(10):1773-1791, 2017.

[7] Tingshu Hu and Franco Blanchini. Non-conservative matrix inequality conditions for stability/stabilizability of linear differential inclusions. Automatica, 46(1):190-196, 2010.

[8] Alexandre Kruszewski, R Wang, and Thierry-Marie Guerra. Nonquadratic stabilization conditions for a class of uncertain nonlinear discrete time TS fuzzy models: A new approach. IEEE Trans. Automatic Control, 53(2):606-611, 2008.

[9] J. W. Lee. On uniform stabilization of discrete-time linear parameter-varying control systems. IEEE Transactions on Automatic Control, 51(10):1714-1721, Oct 2006.

[10] Ji-Woong Lee and Geir E Dullerud. Uniform stabilization of discrete-time switched and Markovian jump linear systems. Automatica, 42(2):205-218, 2006.

[11] Panagiotis Patrinos, Pantelis Sopasakis, Haralambos Sarimveis, and Alberto Bemporad. Stochastic model predictive control for constrained discrete-time Markovian switching systems. Automatica, 50(10):2504-2514, 2014.

[12] Antonio Sala, Manuel Hernández-Mejías, and Carlos Ariño. Stable receding-horizon scenario predictive control for Markov-jump linear systems. Automatica, 86:121 - 128, 2017.

[13] Jeff S Shamma. An overview of LPV systems. In J. Mohammadpour and C.W. Scherer, editors, Control of linear parameter varying systems with applications, pages 326. Springer, 2012. 
[14] Eduardo S. Tognetti, Ricardo C.L.F. Oliveira, and Pedro L.D. Peres. $\mathcal{H}_{\infty}$ and $\mathcal{H}_{2}$ nonquadratic stabilisation of discretetime Takagi-Sugeno systems based on multi-instant fuzzy Lyapunov functions. Int. J. Systems Science, 46(1):76-87, 2015.

[15] Weiming Xiang. Parameter-memorized Lyapunov functions for discrete-time systems with time-varying parametric uncertainties. Automatica, 87:450 - 454, 2018. 